\title{
Launch Cost Analysis and Optimization Based on Analysis of Space System Characteristics*
}

\author{
Qin Xu, ${ }^{1 \dagger \dagger}$ Peter HollingsworTh, ${ }^{2)}$ and Katharine $\mathrm{SMITH}^{2)}$ \\ ${ }^{1)}$ Center for Assessment and Demonstration Research, Academy of Military Science, Beijing 100091, China \\ ${ }^{2)}$ School of Mechanical, Aerospace and Civil Engineering, The University of Manchester, Manchester M13 9PL, United Kingdom
}

\begin{abstract}
The rapid developments in micro-technologies and the introduction of modularity and standardization into system designs, present significant opportunities for cost reduction in the design and development of satellite systems. However, the high cost of space launch has become a major hindrance to capitalizing on these opportunities. Therefore, seeking appropriate launch opportunities and reducing launch costs might contribute to further growth of the space market. This paper focuses on the analysis of dedicated launch costs factoring in the effect of launch reliability, which in return, can enable the optimization of system designs. Applying a value-centric architecture, system characteristic space is introduced as the design space to define the characteristics of different systems. Based on our launch vehicle database, the launch cost and reliability of different families of launch vehicles are investigated, where the reliability is calculated using a modified two-level Bayesian analysis. The factors of launch cost and reliability are subsequently integrated into the expected launch cost, acting as the objective function for the analysis and optimization process associated with the manufacturing cost of satellites. Through reviewing and redesigning a few classical launch cases, the effectiveness and applicability of the design architecture proposed are validated.
\end{abstract}

Key Words: Conceptual Design, Dedicated Launch Cost, Value-centric Design, System Characteristic Space

\section{Introduction}

Due to the rapid development of micro-technologies and the introduction of modularity, a boom in the utilization of small satellites has been witnessed in the past two decades, driving growth in the space market. As shown in Fig. 1, the Union of Concerned Scientists (UCS) ${ }^{1)}$ revealed that the number of small satellites launched has continued to increase sharply since 2012, contributing to the constant increase in the total number of satellites launched. The State of the Satellite Industry Report $2016^{2)}$ also pointed out the continued and growing global interest and expenditure in inexpensive platforms.

Since commercial off-the-shelf (COTS) products have lowered the threshold of spacecraft design and development, satellite manufacturers are realizing significant opportunities for cost reduction. However, the high cost of launch seems to be in opposition to this trend. For instance, developing a typical small satellite costs roughly $\$ 10,000$ per kg, ${ }^{3)}$ while the dedicated launch cost can be much more expensive (e.g., almost double for the European small launch vehicle Vega). ${ }^{4}$ Consequently, seeking an appropriate launch opportunity and reducing launch cost might lead to further growth of the space market.

The current launch opportunities are divided into three categories: dedicated, rideshare, and piggyback launches. ${ }^{5)}$

C 2019 The Japan Society for Aeronautical and Space Sciences

*Presented at the 31st International Symposium on Space Technology and Science, 3-9 June, 2017, Matsuyama, Japan.

Received 22 June 2017; final revision received 12 April 2018; accepted for publication 13 November 2018 .

†Corresponding author, xuqin08@nudt.edu.cn
Since the latter two options involve too much uncertainty beyond the technical level, the research efforts of this paper focus on the dedicated launch cost analysis and optimization for both small and large space systems. It is worth noting that this approach could also be further adapted for piggyback and rideshare launches if the corresponding data were available.

The remainder of this paper is organized as follows. In Section 2, "value-centric design architecture" is introduced to describe the general process for the quantitative design, analysis, and optimization of space system launch cost. Under this design architecture, the system characteristic space, consisting of the degree of duplication, fractionation, and derivation, is presented in Section 3 as the design space to define the characteristics of different systems and enable the optimization of system designs. Based upon our launch

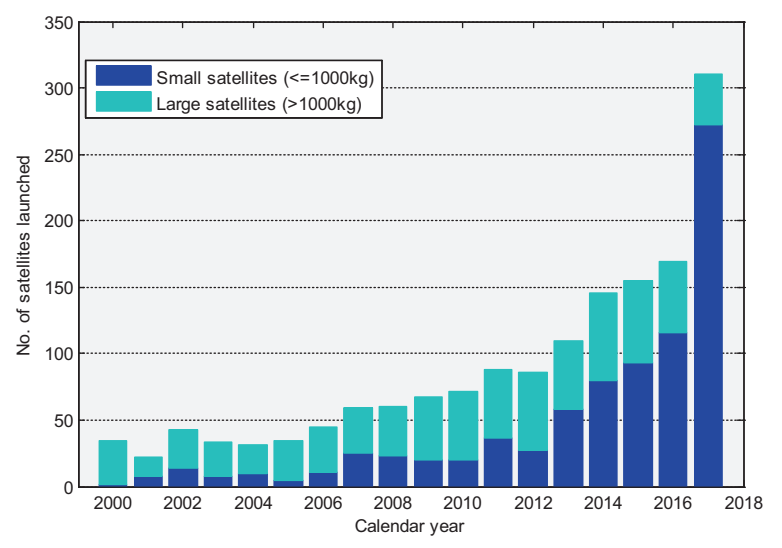

Fig. 1. Recent launch history of the satellites recorded in the UCS satellite database. ${ }^{1)}$ 
vehicle database, Section 4 models and analyses the launch cost and reliability of various families of launch vehicles. Finally, the expected development and deployment cost is proposed as the objective function to enable the optimization process, with the case studies shown in Section 5 and the conclusions presented in Section 6.

\section{Value-centric Design Architecture}

To enable the quantitative analysis and optimization of the system design using both traditional and innovative concepts and technologies, a value-centric design architecture based on system characteristic space is presented.

The basic value flow process in the architecture is shown in Fig. 2. Overall, system value is accumulated from the bottom to the top. It reflects certain design characteristics of space systems. At the subsystem level (System level 3), the value within each subsystem is generated from subsystem properties, such as mass, size, and reliability. The subsystem value is subsequently integrated at the spacecraft level (System level 2). The integrated spacecraft value also influences the value characteristic of the corresponding launch activities (System level 1). Finally, the value of the three system levels is synthesized into the overall system value.

Specifically, in this research, the system property of concern is mass and the corresponding value is the expected launch cost. Through exploring the feasible domain of design variables, namely, different system designs, the analysis and optimization of the launch cost of a space system can be achieved.

Under the value-centric design architecture proposed by Collopy, ${ }^{6}$ ) the specific approaches and techniques of launch cost analysis (Fig. 3) are described as follows.

(1) Elaborate. Through the elaboration process, formulation of the system characteristic space and the overall system configuration definition are established, as well as their transformation relationship.

(2) Analyze. In the analysis process, the system configuration characteristics such as duplication and distribution are analyzed to identify the integration philosophy of the mass properties.

(3) Evaluate. The evaluation process integrates the launch cost value utilizing the appropriate system value models to determine whether or not system requirements are met.

(4) Optimize. In the optimization process, an appropriate optimization algorithm is applied to reach the best solution by quantifying launch cost as the objective function.

\section{System Characteristic Space}

The system characteristic space, ${ }^{7)}$ consisting of duplication, fractionation, and derivation, is introduced to capture and reflect the configuration characteristics of different system designs. Through the system characteristic space proposed, the mapping relationship between a set of conceptual designs and the corresponding system characteristics is established. This enables the quantitative analysis and optimi-

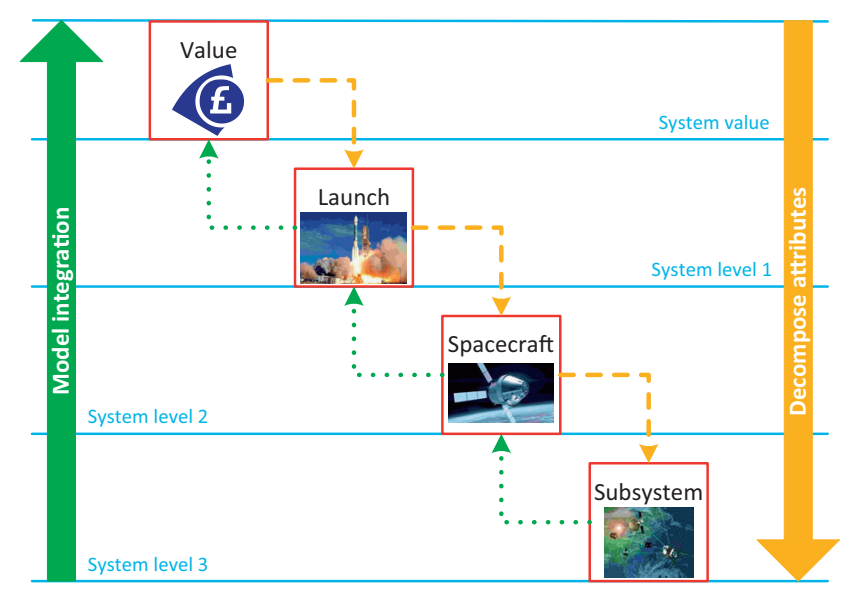

Fig. 2. Value flow diagram.

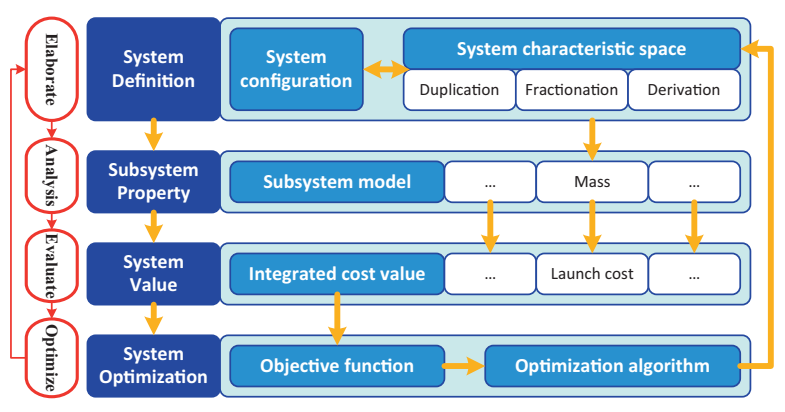

Fig. 3. Value-centric design architecture.

zation of space system design under the value-centric architecture.

The system characteristics are divided into two dimensions: homogeneous and heterogeneous degrees. The homogeneous degree is mathematically defined as the number of identical or near identical components, subsystems, or satellites in a space system; namely degree of duplication. Duplication is widely used to realize an ambitious function or maintain the function in case of unpredictable risks or failures. The heterogeneous degree is on the opposite side, which defines the number of components, subsystems, or satellites performing different functions; namely degree of fractionation. In other words, fractionation describes the spatial distribution of the major functionalities of a space system. In the domain of time, the components, subsystems, or satellites to be designed and produced might be a derivative from previous ones in some ways, and such a heritage factor is defined as degree of derivation. A higher derivative degree generally implies a more mature design, or specifically, a more reliable system.

The detailed descriptions and formulation of the system characteristic space can be found in our previous research. ${ }^{7)}$

\section{Expected Launch Cost Analysis}

Before performing the launch cost analysis, the launch vehicle database is established to provide the data for the modeling of launch reliability and cost. The launch reliability is modeled utilizing two levels of Bayesian analysis, while 
the cost is estimated for a dedicated launch. The estimate of launch reliability also influences the manufacturing cost, since launch failures might result in producing multiple copies of satellites. The reliability and cost information are later integrated into the expected launch cost, which shows the statistical expectation of the cost to ensure inserting a satellite into orbit at given confidence level. The expected launch cost can be used as an objective function for the optimization process associated with the manufacturing cost of satellites.

\subsection{Launch data}

For this research, the launch vehicle database established in our previous research ${ }^{8)}$ was updated to April 2018 based on online space launch reports. ${ }^{9-11)}$ The launch vehicle data is listed in Table 1 and Table 2 for active and retired vehicles, respectively. All of the launch vehicles are categorized into small ( $<5000 \mathrm{lb},<2268 \mathrm{~kg})$, medium $(<12000 \mathrm{lb}$, $<5443 \mathrm{~kg}$ ), intermediate $(<25000 \mathrm{lb},<11340 \mathrm{~kg})$, and heavy ( $\geq 25000 \mathrm{lb}, \geq 11340 \mathrm{~kg}$ ) classes, according to their launch capabilities in pounds to Low Earth Orbit (LEO). ${ }^{12)}$
It is worth noting that the data of launch costs are the average costs of launch campaigns for each launch vehicle family. This may exclude the costs such as insurance and safety management. However, this is only a tiny proportion of the total launch costs, and therefore does not affect the significance of this study.

\subsection{Bayesian analysis of launch reliability}

In the database established, all of launch vehicles are categorized into different families. In each family, the launch vehicles are related and derivative to a great extent, due to the similar levels of individual technologies utilized. For those utilizing different or advanced technologies, a new family is generated, e.g., Atlas 400s and 500s. Thus, the record of launch history for different families of launch vehicles can be used to analyze their launch reliability.

Assuming the probability of successes of a launch vehicle family remains constant for every experiment, the space launch activities can be modeled as a series of Bernoulli trials $^{13)}$ defined as random experiments with exactly two out-

Table 1. Launch data for active launch vehicle families.*

\begin{tabular}{|c|c|c|c|c|c|c|c|c|c|}
\hline Launch vehicle family & Country & Mass class & $\begin{array}{c}\text { Capability } \\
\text { to LEO } \\
(\mathrm{kg})\end{array}$ & $\begin{array}{c}\text { Capability } \\
\text { to GTO } \\
(\mathrm{kg})\end{array}$ & $\begin{array}{c}\text { Payload } \\
\text { length } \\
(\mathrm{m})\end{array}$ & $\begin{array}{c}\text { Payload } \\
\text { diameter } \\
(\mathrm{m})\end{array}$ & $\begin{array}{l}\text { Launch cost } \\
\text { (FY2010\$M) }\end{array}$ & $\begin{array}{c}\text { Number } \\
\text { of } \\
\text { successes }\end{array}$ & $\begin{array}{c}\text { Number } \\
\text { of } \\
\text { failures }\end{array}$ \\
\hline Ariane $5 \mathrm{G} \& 5 \mathrm{E}$ & Europe & Heavy & 21000 & 6800 & 16.19 & 4.57 & 175.99 & 92 & 5 \\
\hline Atlas 5 401, 411, 421, 431 & USA & Heavy & 15700 & 7700 & 10.31 & 3.75 & 152.00 & 51 & 1 \\
\hline Atlas 5 501, 521, 531, 541, 551 & USA & Heavy & 20520 & 8900 & 12.92 & 4.57 & 172.50 & 23 & 0 \\
\hline Delta $26000 \& 7000$ & USA & Medium & 5144 & 1800 & 6.83 & 2.74 & 74.91 & 152 & 2 \\
\hline Delta $4 \mathrm{M}, 4 \mathrm{M}+, 4 \mathrm{H}$ & USA & Heavy & 22560 & 13130 & 15.71 & 4.57 & 215.00 & 35 & 1 \\
\hline Dnepr & Russia & Medium & 4400 & 0 & 4.20 & 2.70 & 20.43 & 21 & 1 \\
\hline Epsilon & Japan & Small & 1200 & 0 & 5.39 & 2.12 & 38.00 & 3 & 0 \\
\hline Falcon 9 V1.0, 1.1, FT & USA & Heavy & 22800 & 8300 & 11.00 & 4.60 & 56.22 & 45 & 3 \\
\hline Falcon Heavy & USA & Heavy & 63800 & 26700 & 13.90 & 5.20 & 81.61 & 1 & 0 \\
\hline GSLV & India & Medium & 5000 & 2350 & 7.30 & 3.05 & 44.00 & 6 & 5 \\
\hline $\mathrm{H}-2 \& 2 \mathrm{~A}$ & Japan & Heavy & 11730 & 5000 & 10.23 & 3.70 & 101.25 & 41 & 3 \\
\hline H-2B & Japan & Heavy & 16500 & 8000 & 9.12 & 4.60 & 142.42 & 6 & 0 \\
\hline Kosmos 3M & Russia & Small & 1500 & 0 & 4.72 & 2.40 & 18.39 & 424 & 22 \\
\hline $\mathrm{KZ}$ & China & Small & 300 & 0 & 1.40 & 1.20 & 2.61 & 3 & 0 \\
\hline Long March 2C \& 2D & China & Medium & 3500 & 1000 & 5.00 & 3.00 & 20.49 & 81 & 2 \\
\hline Long March 2E \& 2F & China & Intermedium & 8400 & 3500 & 6.54 & 3.80 & 68.10 & 18 & 2 \\
\hline Long March 3A \& 3C & China & Intermedium & 9100 & 3800 & 5.25 & 3.00 & 102.45 & 40 & 0 \\
\hline Long March 3B & China & Heavy & 13600 & 5200 & 6.85 & 3.85 & 81.72 & 41 & 3 \\
\hline Long March 4A, 4B, 4C & China & Medium & 4200 & 1500 & 6.50 & 3.35 & 47.81 & 50 & 2 \\
\hline Long March 5 & China & Heavy & 23000 & 13000 & 10.25 & 4.50 & 150.10 & 1 & 1 \\
\hline Long March 6 & China & Small & 1500 & 0 & 4.60 & 2.20 & 13.05 & 2 & 0 \\
\hline Long March 7 & China & Heavy & 13500 & 5500 & 6.85 & 3.80 & 87.45 & 2 & 0 \\
\hline Long March 11 & China & Small & 700 & 0 & 2.00 & 1.60 & 6.09 & 3 & 0 \\
\hline Minotaur I \& IV & USA & Small & 1735 & 0 & 5.71 & 2.04 & 45.96 & 17 & 0 \\
\hline Minotaur V & USA & Small & 630 & 630 & 4.02 & 2.04 & 45.96 & 1 & 0 \\
\hline Pegasus \& Pegasus XL & USA & Small & 443 & 0 & 2.12 & 1.16 & 18.45 & 37 & 6 \\
\hline Proton $\mathrm{K} \& \mathrm{M}$ & Russia & Heavy & 21000 & 5500 & 9.86 & 3.86 & 141.15 & 367 & 46 \\
\hline PSLV, PSLV-CA, PSLV-XL & India & Medium & 3800 & 1300 & 5.40 & 2.90 & 20.11 & 39 & 3 \\
\hline Rockot & Russia & Small & 1950 & 0 & 6.21 & 2.38 & 18.45 & 27 & 3 \\
\hline Shavit $1 \& 2$ & Israel & Small & 800 & 0 & 2.84 & 1.3 & 20.49 & 8 & 2 \\
\hline Shtil 1 & Russia & Small & 140 & 0 & 1.47 & 0.63 & 2.20 & 2 & 0 \\
\hline Soyuz 2.1 & Russia & Intermedium & 8200 & 3250 & 9.51 & 3.80 & 54.65 & 65 & 5 \\
\hline Soyuz U & Russia & Intermedium & 7000 & 1660 & 9.00 & 2.85 & 54.65 & 764 & 21 \\
\hline Start-1 & Russia & Small & 632 & 0 & 2.31 & 1.24 & 12.29 & 6 & 0 \\
\hline Strela & Russia & Small & 1560 & 0 & 2.95 & 2.20 & 14.34 & 3 & 0 \\
\hline Taurus \& Taurus XL & USA & Small & 1590 & 557 & 5.71 & 2.04 & 45.26 & 7 & 3 \\
\hline Vega & Europe & Small & 1963 & 0 & 6.30 & 2.30 & 35.00 & 11 & 0 \\
\hline Volna & Russia & Small & 140 & 0 & 1.25 & 0.82 & 1.57 & 2 & 3 \\
\hline Zenit 3 SL, SLB, SLBF & Russia & Heavy & 15876 & 6066 & 8.53 & 3.75 & 115.77 & 42 & 4 \\
\hline
\end{tabular}

${ }^{*}$ Small, medium, intermedium, and heavy launch vehicles are identified by their payload capabilities in kg to LEO (<2268, $\left.<5443,<11340, \geq 11340\right)$. 
Trans. Japan Soc. Aero. Space Sci., Vol. 62, No. 4, 2019

Table 2. Launch data for retired launch vehicle families.

\begin{tabular}{|c|c|c|c|c|c|c|c|c|c|}
\hline Launch vehicle family & Country & Mass class & $\begin{array}{c}\text { Capability } \\
\text { to LEO } \\
(\mathrm{kg})\end{array}$ & $\begin{array}{c}\text { Capability } \\
\text { to GTO } \\
(\mathrm{kg})\end{array}$ & $\begin{array}{l}\text { Payload } \\
\text { length } \\
(\mathrm{m})\end{array}$ & $\begin{array}{l}\text { Payload } \\
\text { diameter } \\
(\mathrm{m})\end{array}$ & $\begin{array}{l}\text { Launch cost } \\
(\mathrm{FY} 2010 \$ \mathrm{M})\end{array}$ & $\begin{array}{c}\text { Number } \\
\text { of } \\
\text { successes }\end{array}$ & $\begin{array}{c}\text { Number } \\
\text { of } \\
\text { failures }\end{array}$ \\
\hline Athena $1 \& 2$ & USA & Small & 2065 & 0 & 4.29 & 2.05 & 32.69 & 5 & 2 \\
\hline Ariane 4 & Europe & Intermedium & 10200 & 4790 & 11.12 & 3.65 & 153.23 & 113 & 3 \\
\hline Atlas 2, 2A, 2AS & USA & Intermedium & 8618 & 3719 & 9.39 & 3.75 & 132.80 & 63 & 0 \\
\hline Atlas 3 & USA & Intermedium & 10759 & 4119 & 10.31 & 3.75 & 141.62 & 6 & 0 \\
\hline Cyclone $2 \& 3$ & Russia & Medium & 4100 & 0 & 6.60 & 2.40 & 28.28 & 219 & 9 \\
\hline Delta 2000 & USA & Small & 1860 & 724 & 4.93 & 2.18 & 57.90 & 43 & 1 \\
\hline Delta 38000 & USA & Intermedium & 8290 & 3810 & 9.93 & 3.75 & 122.94 & 1 & 2 \\
\hline Delta 3000, 4000, 5000 & USA & Medium & 3848 & 1405 & 6.12 & 2.54 & 67.85 & 38 & 3 \\
\hline Falcon 1 & USA & Small & 668 & 0 & 2.79 & 1.37 & 6.95 & 2 & 3 \\
\hline Long March 2A & China & Small & 2000 & 0 & 5.00 & 3.00 & 28.80 & 3 & 1 \\
\hline Molniya-M & Russia & Medium & 3700 & 0 & 4.04 & 2.35 & 47.81 & 276 & 21 \\
\hline M-V & Japan & Small & 1900 & 1280 & 6.03 & 2.20 & 71.65 & 6 & 1 \\
\hline Soyuz & Russia & Intermedium & 7000 & 1350 & 5.86 & 3.43 & 51.08 & 30 & 2 \\
\hline Space Shuttle & USA & Heavy & 28803 & 5900 & 18.3 & 4.57 & 408.60 & 133 & 2 \\
\hline Titan 2 & USA & Small & 1900 & 0 & 6.65 & 2.83 & 47.81 & 13 & 0 \\
\hline $\operatorname{Titan} 3 \mathrm{~A} \&$ \&B & USA & Medium & 3300 & 0 & 6.75 & 2.83 & 49.45 & 66 & 6 \\
\hline Titan 3C, 3D, 3E, 34D & USA & Heavy & 14515 & 5000 & 8.27 & 2.83 & 250.93 & 71 & 9 \\
\hline Titan 4A \& 4B & USA & Heavy & 21600 & 5760 & 18.23 & 4.57 & 546.38 & 35 & 4 \\
\hline VLS-1 & Brazil & Small & 380 & 0 & 2.05 & 1.10 & 9.42 & 0 & 3 \\
\hline Zenit 2 & Russia & Heavy & 13740 & 0 & 12.24 & 3.40 & 57.89 & 30 & 8 \\
\hline
\end{tabular}

comes: success or failure. Our work is to achieve the best estimate of this probability of success.

One of the biggest challenges in the modeling of space launch activities, distinguishing it from other Bernoulli trials such as coin-toss problems, is the limited sample size. This may increase the inaccuracy of the estimate. The Bayesian method is proposed to overcome this problem ${ }^{14)}$ as it incorporates prior knowledge of the known launch vehicles into the records of the observed launches of the launch vehicles in question, shown in Eq. (1).

$$
f_{A \mid D}(a \mid s, f)=\frac{f_{D \mid A}(s, f \mid a) f_{A}(a)}{\int f_{D \mid A}(s, f \mid x) f_{A}(x) d x}
$$

Where, $A$ is the probability of successful launches given $s$ successes and $f$ failures in the past $t=s+f$ trials. The probability density function $f_{A}(a)$ is the prior distribution, representing the state of knowledge about a given launch vehicle prior to any of the $t$ launch trials. The likelihood function $f_{D \mid A}(s, f \mid a)$ denotes the probability of the observed data $D$, namely, achieving $s$ successes and $f$ failures in $t$ launch attempts. The result of the calculation $f_{A \mid D}(a \mid s, f)$ is the posterior distribution, which updates the previous knowledge with the realized launch data.

In previous research, ${ }^{15)}$ Howard derived a general form of Bayesian probability and demonstrated that the mean of the posterior distribution is the optimal estimate of the probability of successes. Based upon these mathematical fundamentals, Guikema and Pate-Cornell proposed using beta distribution as the conjugate prior distribution for modeling and analyzing the reliability of launch vehicles. ${ }^{16}$ )

$$
f_{A}\left(a \mid s_{0}, f_{0}\right)=\frac{\Gamma\left(s_{0}+f_{0}\right)}{\Gamma\left(s_{0}\right) \Gamma\left(f_{0}\right)} p^{s_{0}-1}(1-p)^{f_{0}-1}
$$

Where, $\Gamma(n)$ is the gamma function with parameter $n$.

$$
\Gamma(n)=\int_{0}^{\infty} t^{n-1} e^{-t} d t
$$

The mean and variance of the beta distribution can be presented by the parameters $s$ and $f$.

$$
\begin{gathered}
\bar{p}=\frac{s}{s+f} \\
\sigma=\frac{s f}{(s+f)^{2}(s+f+1)}
\end{gathered}
$$

If the updated observed data of $s$ successes and $f$ failures can be considered as the Bernoulli process, the posterior distribution is also a beta distribution with the parameters $s_{0}+s$ and $f_{0}+f$.

$$
\begin{aligned}
f_{A \mid D}(a \mid s, f)= & \frac{\Gamma\left(s_{0}+s+f_{0}+f\right)}{\Gamma\left(s_{0}+s\right) \Gamma\left(f_{0}+f\right)} p^{s_{0}+s-1} \\
& \cdot(1-p)^{f_{0}+f-1}
\end{aligned}
$$

The two levels of Bayesian analysis proposed by Guikema and Pate-Cornell ${ }^{16)}$ are adopted in this paper for the reliability analysis of different types of launch vehicles. The first level applies the uniform distribution for the prior distribution of the Bayesian method. In this case, we assume that we know nothing about the reliability of these launch vehicles in advance. This level is appropriate for those launch vehicles with sufficient updating data, so that the prior distribution will have little influence on the posterior distribution, since the data dominates the updating process.

We fit the second-level prior distributions by combining the means of the first-level posterior distributions for all the launch vehicles except the one in question. This incorporates all of the experience of known launch vehicles. In this case, we assume that the probability of success for emerging launch vehicles is similar to previous ones, as much of the lessons learned in previous generations have been widely 
Trans. Japan Soc. Aero. Space Sci., Vol. 62, No. 4, 2019

Table 3. Means and variances of the posterior probability distribution function for active launch vehicle families.*

\begin{tabular}{|c|c|c|c|c|c|c|}
\hline \multirow{2}{*}{ Launch vehicle family } & \multirow{2}{*}{ Successes/Attempts } & \multirow{2}{*}{ Successful rate $(\%)$} & \multicolumn{2}{|c|}{ First-level posterior } & \multicolumn{2}{|c|}{ Second-level posterior } \\
\hline & & & Means (\%) & Variances $(\%)$ & Means (\%) & Variances $(\%)$ \\
\hline Ariane $5 \mathrm{G} \& 5 \mathrm{E}$ & $92 / 97$ & 94.85 & 93.94 & 0.06 & 94.35 & 0.05 \\
\hline Atlas 5 401, 411, 421, 431 & $51 / 52$ & 98.08 & 96.30 & 0.06 & 96.93 & 0.05 \\
\hline Atlas $5501,521,531,541,551$ & $23 / 23$ & 100.00 & 96.00 & 0.15 & 97.35 & 0.09 \\
\hline Delta $26000 \& 7000$ & $152 / 154$ & 98.70 & 98.08 & 0.01 & 98.28 & 0.01 \\
\hline Delta $4 \mathrm{M}, 4 \mathrm{M}+, 4 \mathrm{H}$ & $35 / 36$ & 97.22 & 94.74 & 0.13 & 95.72 & 0.10 \\
\hline Dnepr & $21 / 22$ & 95.45 & 91.67 & 0.31 & 93.44 & 0.23 \\
\hline Epsilon & $3 / 3$ & 100.00 & 80.00 & 2.67 & 90.12 & 1.12 \\
\hline Falcon 9 V1.0, 1.1, FT & $45 / 48$ & 93.75 & 92.00 & 0.14 & 92.87 & 0.12 \\
\hline Falcon Heavy & $1 / 1$ & 100.00 & 66.67 & 5.56 & 86.29 & 1.98 \\
\hline GSLV & $6 / 11$ & 54.55 & 53.85 & 1.78 & 62.29 & 1.46 \\
\hline $\mathrm{H}-2 \& 2 \mathrm{~A}$ & $41 / 44$ & 93.18 & 91.30 & 0.17 & 92.28 & 0.15 \\
\hline $\mathrm{H}-2 \mathrm{~B}$ & $6 / 6$ & 100.00 & 87.50 & 1.22 & 93.01 & 0.59 \\
\hline Kosmos 3M & $424 / 446$ & 95.07 & 94.87 & 0.01 & 94.95 & 0.01 \\
\hline $\mathrm{KZ}$ & $3 / 3$ & 100.00 & 80.00 & 2.67 & 90.12 & 1.12 \\
\hline Long March 2C \& 2D & $81 / 83$ & 97.59 & 96.47 & 0.04 & 96.88 & 0.03 \\
\hline Long March 2E \& 2F & $18 / 20$ & 90.00 & 86.36 & 0.51 & 88.76 & 0.40 \\
\hline Long March 3A \& 3C & $40 / 40$ & 100.00 & 97.62 & 0.05 & 98.36 & 0.04 \\
\hline Long March 3B & $41 / 44$ & 93.18 & 91.30 & 0.17 & 92.28 & 0.15 \\
\hline Long March 4A, 4B, 4C & $50 / 52$ & 96.15 & 94.44 & 0.10 & 95.16 & 0.08 \\
\hline Long March 5 & $1 / 2$ & 50.00 & 50.00 & 5.00 & 72.37 & 2.79 \\
\hline Long March 6 & $2 / 2$ & 100.00 & 75.00 & 3.75 & 88.52 & 1.46 \\
\hline Long March 7 & $2 / 2$ & 100.00 & 75.00 & 3.75 & 88.52 & 1.46 \\
\hline Long March 11 & $3 / 3$ & 100.00 & 80.00 & 2.67 & 90.12 & 1.12 \\
\hline Minotaur I \& IV & $17 / 17$ & 100.00 & 94.74 & 0.25 & 96.61 & 0.15 \\
\hline Minotaur V & $1 / 1$ & 100.00 & 66.67 & 5.56 & 86.29 & 1.98 \\
\hline Pegasus \& Pegasus XL & $37 / 43$ & 86.05 & 84.44 & 0.29 & 85.75 & 0.25 \\
\hline Proton $\mathrm{K} \& \mathrm{M}$ & $367 / 413$ & 88.86 & 88.67 & 0.02 & 88.80 & 0.02 \\
\hline PSLV, PSLV-CA, PSLV-XL & $39 / 42$ & 92.86 & 90.91 & 0.18 & 91.95 & 0.16 \\
\hline Rockot & $27 / 30$ & 90.00 & 87.50 & 0.33 & 89.12 & 0.28 \\
\hline Shavit $1 \& 2$ & $8 / 10$ & 80.00 & 75.00 & 1.44 & 80.76 & 1.04 \\
\hline Shtil 1 & $2 / 2$ & 100.00 & 75.00 & 3.75 & 88.52 & 1.46 \\
\hline Soyuz 2.1 & $65 / 70$ & 92.86 & 91.67 & 0.10 & 92.29 & 0.09 \\
\hline Soyuz U & $764 / 785$ & 97.32 & 97.20 & 0.00 & 97.25 & 0.00 \\
\hline Start-1 & $6 / 6$ & 100.00 & 87.50 & 1.22 & 93.01 & 0.59 \\
\hline Strela & $3 / 3$ & 100.00 & 80.00 & 2.67 & 90.12 & 1.12 \\
\hline Taurus \& Taurus XL & $7 / 10$ & 70.00 & 66.67 & 1.71 & 73.65 & 1.30 \\
\hline Vega & $11 / 11$ & 100.00 & 92.31 & 0.51 & 95.29 & 0.28 \\
\hline Volna & $2 / 5$ & 40.00 & 42.86 & 3.06 & 60.03 & 2.33 \\
\hline Zenit 3 SL, SLB, SLBF & $42 / 46$ & 91.30 & 89.58 & 0.19 & 90.60 & 0.17 \\
\hline
\end{tabular}

*The means and variances of the posterior probability to be used for each launch vehicle family are highlighted in bold.

shared. The recorded data of all the other launch vehicles acts as the prior knowledge of launch activities, while the data of the launch vehicle to be investigated is used for the updating process. Therefore, this level of the Bayesian analysis can compensate for the lack of information about new launch vehicles or ones with limited launch trials. In addition, removing the information of the vehicle to be investigated in the prior distribution can solve the redundancy problem existing in the original second-level Bayesian analysis proposed by Guikema and Pate-Cornell. ${ }^{16)}$

The results of the two levels of Bayesian analysis for different launch vehicle families are summarized in Table 3, with the corresponding successful rates as the references. Overall, the Delta 2 family is the most reliable current launch vehicle, while the reliability estimate of the Soyuz $U$ family exhibits the lowest uncertainty or variance as a result of the shear number of launches. Among the launch vehicles with limited trials, the Minotaur and Vega families have the highest expected reliability.
As stated above, different levels of reliability analysis are appropriate for different vehicles. In this research, we assume that the launch vehicles with at least 20 launches are considered to have comparatively sufficient updating data, thus are considered using only first-level analysis. On the contrary, the launch vehicles with less than 20 launches are classified as relatively novel or infrequently used, and considered using second-level analysis. Furthermore, the probability to be used for each launch vehicle family is highlighted in bold in Table 3.

Therefore, the posterior distributions of the Bayesian probability for different launch vehicle families are shown in Figs. 4 and 5, respectively. In both figures, the $x$-axis denotes the value of launch reliability and the $y$-axis shows the corresponding probability density.

As shown in Fig. 5, the absence of the intermedium class indicates that all of the active launch vehicles in this class are appropriate for first-level analysis. In other words, most novel launch vehicles are in the smaller classes, while the 


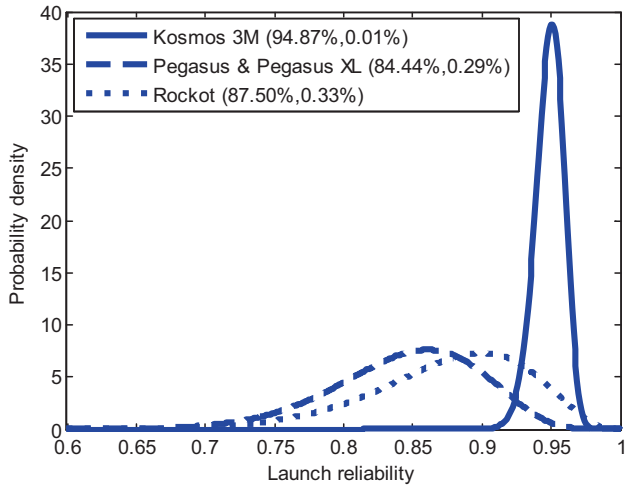

(a) Small launch vehicles

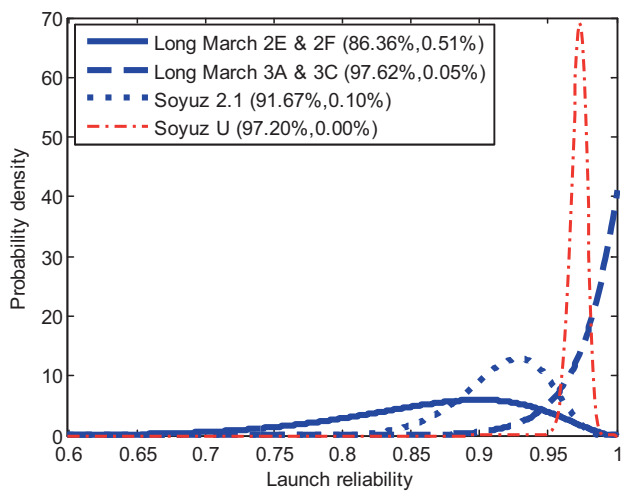

(c) Intermedium launch vehicles

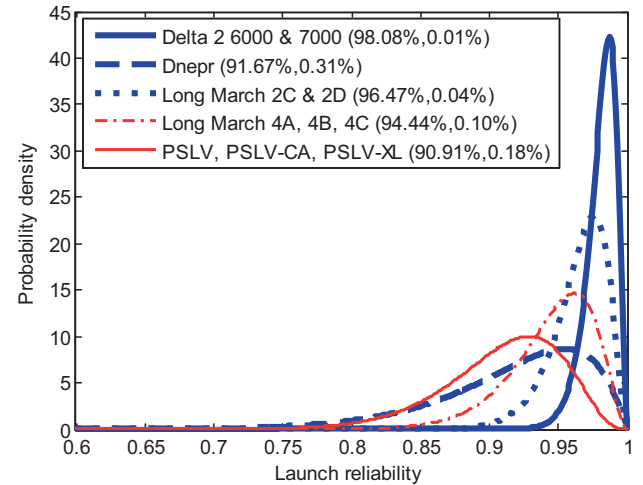

(b) Medium launch vehicles

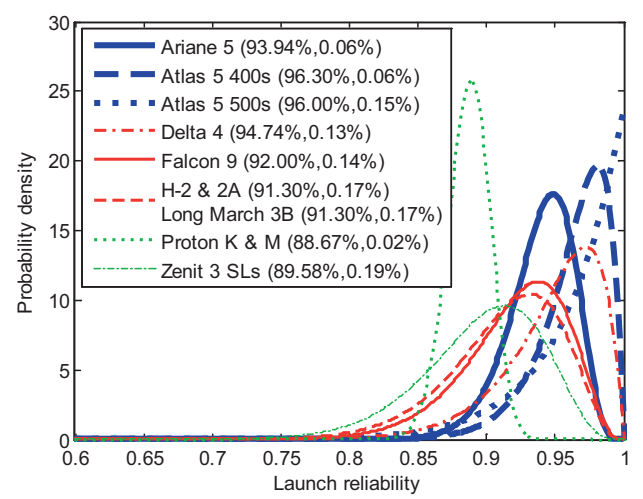

(d) Heavy launch vehicles

Fig. 4. First-level posterior probability function for launch vehicles with at least 20 launch attempts.

other classes are mainly mature launch vehicles. Additionally, it is noteworthy that the launch vehicles lacking capability or cost information (e.g., SS-520) are not included here as additional information is necessary to enable use in launch cost analysis and optimization.

\subsection{Expected launch cost}

Expected launch cost refers to the statistical expectation of the cost of a successful launch; that is, successfully inserting the payload into an effective orbit, which is the combination of launch cost and launch reliability.

$$
c_{e}=n_{e} \cdot c_{l}
$$

Where, $c_{e}$ denotes the proposed expected launch cost, $c_{l}$ is the dedicated launch cost of a single launch attempt, and the number of statistical average launch attempts $n_{e}$ to achieve a successful launch is defined by Eq. (8).

$$
1-(1-r)^{n_{e}} \geq 1-\alpha
$$

Where, $r$ is the reliability of the launch vehicle and $\alpha$ is the significant level or $1-\alpha$ is the confidence level, a measure of how confident we want to be. Thus,

$$
c_{e} \geq c_{l} \cdot \log _{1-r} \alpha
$$

With the unit launch cost and reliability of different launch vehicles as the inputs, the expected launch cost can be obtained, taking $\alpha$ as 0.01 . Therefore, the expected number of launch attempts and the corresponding cost of different launch vehicle families can be calculated utilizing Eq. (9) under a confidence level of $99 \%$. The results are listed in
Table 4. Utilizing this table, the optimal launch strategy can be looked up once the payload mass distribution is determined.

\section{Optimization}

To enable the optimization process of seeking an appropriate launch vehicle, the expected launch cost is used as the objective function associated with the development cost of the satellites to be launched. Having identified the problem as a mixed integer nonlinear programming (MINLP) problem, an appropriate optimization algorithm is adopted. Three classical launch cases are reviewed and redesigned to show the effectiveness and applicability of the method proposed.

\subsection{Optimization techniques}

The optimization process for space system launch cost can be mathematically summarized as a MINLP problem, Eq. (10).

$$
\begin{array}{ll}
\min & J(x) \\
\text { s.t. } & x_{j}^{l} \leq x_{j} \leq x_{j}^{u} \text { : integer } j=1,2, \cdots, n \\
& x=\left[\begin{array}{llll}
x_{1} & x_{2} & \cdots & x_{n}
\end{array}\right]^{\mathrm{T}}
\end{array}
$$

Where, $J(x)$ is the objective function, $n$ is the number of state variables, $x_{j}$ is the state variable, and $x_{i}^{l}$ and $x_{i}^{u}$ are the corresponding lower and upper bounds, respectively.

More specifically, the objective function $J(x)$ is the launch cost under riskin this research, which will be described in detail. The state variable $x$ is the System Sequence Vector pro- 


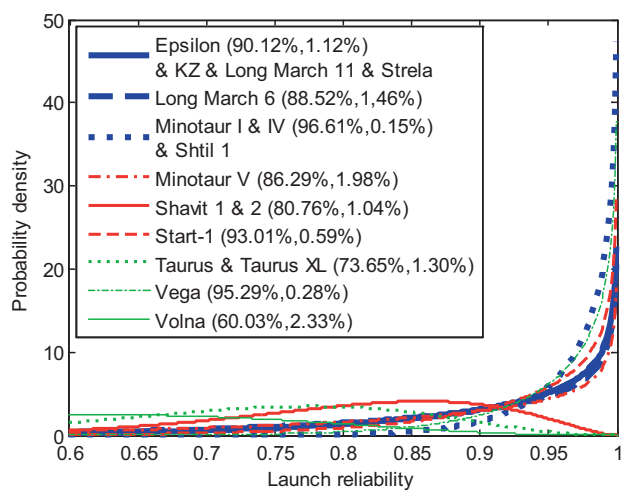

(a) Small launch vehicles

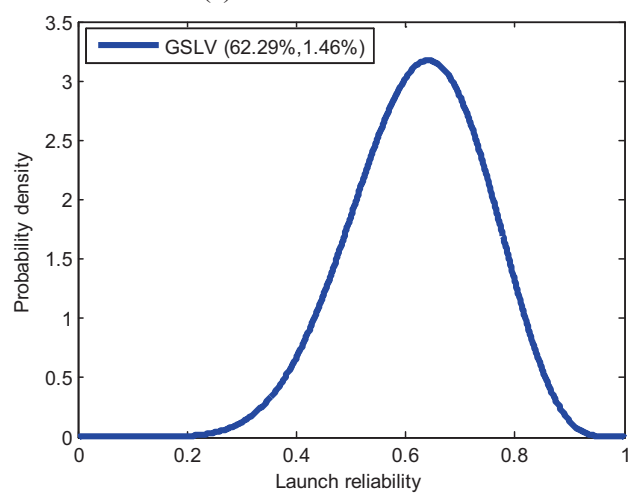

(b) Medium launch vehicles

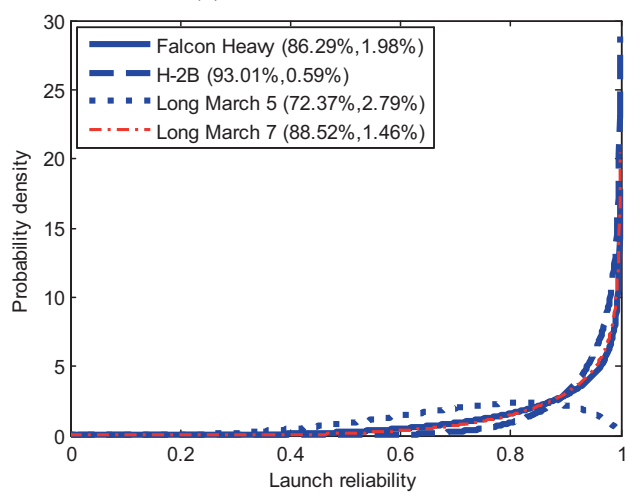

(c) Heavy launch vehicles

Fig. 5. Second-level posterior probability function for launch vehicles with less than 20 launch attempts.

posed in our previous research. ${ }^{7)}$ It is used to define the combinations and permutations of a space system (e.g., a constellation of identical spacecraft or a fractionated spacecraft) when considering duplication and/or fractionation. As the elements of the System Sequence Vector are all integers, ${ }^{7)}$ this optimization can be included in the MINLP problem.

Since MINLP problems have been widely discussed in previous studies, one of the recent findings is adopted here, namely, the genetic algorithm (GA) associated with Laplace Crossover (LX), ${ }^{17)}$ Power Mutation (PM), ${ }^{18)}$ and a truncation technique, which is called the MI-LXPM algorithm by Deep et al. ${ }^{19)}$

\subsection{Objective function}

Adopting the expected launch cost as the objective function for the optimization process is one of the simplest solutions. However, if the design and development cost of space
Table 4. Expected number of launches required to get a $99 \%$ confidence in deploying necessary satellites in orbit and the corresponding cost for active launch vehicle families.

\begin{tabular}{|c|c|c|}
\hline Launch vehicle family & $\begin{array}{l}\text { Expected } \\
\text { launches }\end{array}$ & $\begin{array}{l}\text { Expected cost } \\
(\mathrm{FY} 2010 \$ \mathrm{M})\end{array}$ \\
\hline Ariane $5 \mathrm{G} \& 5 \mathrm{E}$ & 1.64 & 289.10 \\
\hline Atlas 5 401, 411, 421, 431 & 1.40 & 212.38 \\
\hline Atlas 5 501, 521, 531, 541, 551 & 1.43 & 246.79 \\
\hline Delta $26000 \& 7000$ & 1.17 & 87.31 \\
\hline Delta $4 \mathrm{M}, 4 \mathrm{M}+, 4 \mathrm{H}$ & 1.56 & 336.26 \\
\hline Dnepr & 1.85 & 37.86 \\
\hline Epsilon & 2.55 & 96.82 \\
\hline Falcon 9 V1.0, 1.1, FT & 1.82 & 102.51 \\
\hline Falcon Heavy & 3.38 & 275.62 \\
\hline GSLV & 5.52 & 242.72 \\
\hline $\mathrm{H}-2 \& 2 \mathrm{~A}$ & 1.89 & 190.91 \\
\hline $\mathrm{H}-2 \mathrm{~B}$ & 2.07 & 295.15 \\
\hline Kosmos 3M & 1.55 & 28.52 \\
\hline $\mathrm{KZ}$ & 2.50 & 6.52 \\
\hline Long March 2C \& 2D & 1.38 & 28.22 \\
\hline Long March 2E \& 2F & 2.31 & 157.40 \\
\hline Long March 3A \& 3C & 1.23 & 126.23 \\
\hline Long March 3B & 1.89 & 154.09 \\
\hline Long March 4A, 4B, 4C & 1.59 & 76.17 \\
\hline Long March 5 & 5.22 & 783.13 \\
\hline Long March 6 & 2.84 & 37.00 \\
\hline Long March 7 & 2.87 & 251.13 \\
\hline Long March 11 & 2.50 & 15.25 \\
\hline Minotaur I \& IV & 1.49 & 68.58 \\
\hline Minotaur V & 3.37 & 154.98 \\
\hline Pegasus \& Pegasus XL & 2.47 & 45.66 \\
\hline Proton K \& M & 2.11 & 298.43 \\
\hline PSLV, PSLV-CA, PSLV-XL & 1.92 & 38.62 \\
\hline Rockot & 2.21 & 40.86 \\
\hline Shavit $1 \& 2$ & 3.13 & 64.18 \\
\hline Shtil 1 & 2.80 & 6.16 \\
\hline Soyuz 2.1 & 1.85 & 101.28 \\
\hline Soyuz U & 1.29 & 70.35 \\
\hline Start-1 & 2.03 & 24.95 \\
\hline Strela & 2.52 & 36.20 \\
\hline Taurus \& Taurus XL & 3.93 & 177.96 \\
\hline Vega & 1.69 & 59.32 \\
\hline Volna & 6.87 & 10.78 \\
\hline Zenit 3 SL, SLB, SLBF & 2.04 & 235.72 \\
\hline
\end{tabular}

systems is not taken into account, the optimization process is likely to distort the facts. In order to distinguish different risk control requirements, the development and deployment cost is adopted as the objective function of the optimization process, including both the expected launch cost and the cost of the satellites to be launched. Thus,

$$
c_{d}=\left(c_{l}+c_{s}\right) \cdot \log _{1-r} \alpha
$$

where, $c_{d}$ is the expected development and deployment cost, and $c_{s}$ is the cost of the launched satellites.

A learning curve is used to predict the average unit cost of the launched satellites when more than one identical or near identical satellites is produced.

$$
c_{a}=c_{T 1} \cdot n_{l}^{\ln (l) / \ln (2)}
$$

Where, $c_{a}$ is the average unit cost, $c_{T 1}$ is the theoretical first unit production cost, $n_{l}$ is the number of units being built at the same time, and $l$ is the learning curve slope. In the aerospace industry, $l$ is typically $95 \%$. 
Table 5. Cases of dedicated launch cost optimization.

\begin{tabular}{lccc}
\hline Mission & $\begin{array}{c}\text { Actual cost } \\
(\text { FY2010\$M) }\end{array}$ & $\begin{array}{c}\text { Expected cost of } \\
\text { actual launch vehicle } \\
(\text { FY2010\$M) }\end{array}$ & $\begin{array}{c}\text { Optimized } \\
\text { expected cost } \\
(\text { FY2010\$M) }\end{array}$ \\
\hline HST & 408.6 & 492.4 & 336.3 \\
Galileo & 569.0 & 854.4 & 478.0 \\
Flock 3p & $\sim 10.0$ & $35.4^{*}$ & 28.22 \\
\hline
\end{tabular}

*This cost is estimated based on the dedicated launches under risks, while the cost of the piggyback launch that was actually used is given in the actual cost column.

Table 6. Optimal dedicated launch strategies for each case.

\begin{tabular}{lccc}
\hline Mission & $\begin{array}{c}\text { Expected launch } \\
\text { vehicles }\end{array}$ & $\begin{array}{c}\text { Launch } \\
\text { attempts }\end{array}$ & $\begin{array}{c}\text { Number of } \\
\text { satellites per } \\
\text { launch }\end{array}$ \\
\hline HST & Delta 4M, 4M+, 4H & 1 & 1 \\
Galileo & Soyuz U & 5 & 2 \\
Flock 3p & Long March 3A \& 3C & 1 & 4 \\
\hline
\end{tabular}

\subsection{Discussions about optimization results}

The typical launch cases of space systems are reviewed and redesigned by the approach proposed. The results are shown in Table 5 with the specific launch strategies exhibited in Table 6.

The first case is a monolithic spacecraft, the largest and most powerful on-orbit telescope, the Hubble Space Telescope (HST). ${ }^{20)}$ HST weighted $11,866 \mathrm{~kg}$, and its development cost was over $\$ 1,500 \mathrm{M}$ in fiscal year $2010 .^{21)}$ It was deployed by the Space Shuttle Discovery on April 24, 1990 , at an estimated launch cost of more than $\$ 400 \mathrm{M}^{.12)}$

In this case, any launch failure is intolerable since the development cost of the satellite is far more expensive than the launch cost. The risk level of this launch mission needs to be controlled to be as low as possible. Thus, more reliable launch vehicles with adequate launch capability are more appealing. If this mission was implemented by currently available launch vehicles, the Delta 4 would probably be the best solution since the Space Shuttle has been retired.

The second case exhibits the launch strategy optimization of a constellation such as the European Global Navigation Satellite System (GNSS) Galileo. The full operational capability (FOC) of Galileo consists of 14 identical navigation satellites each weighing $733 \mathrm{~kg},{ }^{22)}$ which were launched in six independent launch missions (i.e., the former five missions launched two satellites and the last one launched four satellites). Due to different orbit requirements and manufacturing time, the launch sequence is retained in this case. Therefore, only the launch strategy for each mission is optimized.

The results of this case reveal that the launch missions of Galileo FOC are well-organized, and the optimal strategy only saves $16.0 \%$ of the original cost.

The last is a small satellite constellation case. Flock $3 p$ is a constellation with 88 cubesats each weighting $4.7 \mathrm{~kg}{ }^{23)}$ The mission was performed by PSLV-XL on February 15, $2017,{ }^{24)}$ which launched a record 104 satellites in a single launch attempt.
The reason for the reduction of the launch cost for Flock $3 p$ is that the constellation is the secondary payload of this launch mission. However, the objective of the approach proposed is finding a better dedicated launch strategy. The result shows that the optimal solution saves $20 \%$ of the expected launch cost, but still costs more than the actual mission. This proves the advisability and advantage of rideshare or piggyback launches.

Such a result may have two implications for the space launch market. On one hand, current dedicated launches are not economically comparable to rideshare or piggyback launches in the small satellite market. On the other hand, innovative launch vehicles need to be developed, especially for the small satellite market.

\section{Conclusions}

This research focused on developing a methodology for analyzing and optimizing the dedicated launch cost of different space systems under a value-centric design architecture based on the system characteristic space.

The system characteristic space, consisting of duplication, fractionation, and derivation, is firstly introduced as the design space for analyzing the launch cost. The different designs of a space system can be defined in this space, which enables the integration process of the mass property from the subsystem level into the system level.

Subsequently, system mass property is converted into the launch cost. More specifically, the expected launch cost is adopted as the measurement of system value, which synthesizes the factors of both launch reliability and launch cost. The reliability of different launch vehicles is calculated utilizing a modified two-level Bayesian analysis. The first level is applied for mature launch vehicles with at least 20 launch attempts, while the second level is appropriate for launch vehicles still in the infant phase (e.g., less than 20 launch attempts).

Based upon our launch vehicle database, a value model is established where the expected launch costs of various families of launch vehicles are defined, calculated, and compared under a $99 \%$ confidence level. In order to control the launch risks, the development cost of a space system is introduced to comprise the expected development and deployment cost associated with the expected launch cost. This value is used as the objective function to enable the loop of value flow in the entire architecture.

Having identified the problem proposed as a MINLP problem, an appropriate optimization algorithm is found to reach the solution with the minimum expected launch cost. Three typical cases were investigated and reviewed, and the results validated the effectiveness and the applicability of the valuecentric design approach proposed.

\section{Acknowledgments}

The authors gratefully thank the financial support from the China Scholarship Council (CSC) (201403170413) for this work. 


\section{References}

1) Union of Concerned Scientists: UCS Satellite Database, 2016, Available: http://www.ucsusa.org/nuclear-weapons/space-weapons/ satellite-database\#.V790ZUYrKUk (accessed 2018-04-01)

2) Satellite Industry Association: State of the Satellite Industry Report, Washington, DC, 2016.

3) Delft University of Technology: Small Satellite Projects and Their Cost, 2016, Available: http:/www.lr.tudelft.nl/en/organisation/ departments/space-engineering/space-systems-engineering/expertiseareas/mission-concept-exploration/small-satellite-projects/ (accessed 2016-08-29)

4) British Broadcasting Corporation: Vega Rocket Set for Maiden Voyage, 2012, Available: http://www.bbc.co.uk/news/scienceenvironment-16986043 (accessed 2016-08-29)

5) Crisp, N., Smith, K., and Hollingsworth, P.: Small Satellite Launch to LEO: A Review of Current and Future Launch Systems, Trans. JSASS Aerospace Technology Japan, 12, ists29 (2014), pp. Tf_39-Tf_47.

6) Collopy, P.: Economic-based Distributed Optimal Design, AIAA Space 2001 Conference and Exposition, 2001, pp. 4675.

7) $\mathrm{Xu}, \mathrm{Q}$., Hollingsworth, P. M., and Smith, K. L.: Value-Centric Design Architecture Based on Analysis of Space System Characteristics, Acta Astronautica, 144 (2018), pp. 69-79.

8) Xu, Q., Zhang, M., Hao, Z., Hollingsworth, P., and Smith, K.: Small Satellite Launch Opportunity: Statistical Analysis and Trend Forecast, 67th International Astronautical Congress (IAC), 2016, pp. 1-10.

9) Krebs, G. D.: Gunter's Space Page, 2016, Available: http: //space.skyrocket.de/index.html (accessed 2018-04-01)

10) Wade, M.: Encyclopedia Astronautica, 2016, Available: http: //www.astronautix.com/index.html (accessed 2018-04-01)

11) Kyle, E.: Space Launch Report, 2016, Available: http: //www.spacelaunchreport.com/ (accessed 2018-04-01)

12) Wertz, J. R., Everett, D. F., and Puschell, J. J.: Space Mission Engineering: The New SMAD, Microcosm Press, Hawthorne, CA, 2011.
13) Pillai, S. and Papoulis, A.: Probability, Random Variables and Stochastic Processes, 2nd ed., McGraw-Hill, New York, 2001

14) Scheaffer, R. L. and McClave, J. T.: Probability and Statistics for Engineers, P.W.S.-Kent Publishing Company, Boston, 1990.

15) Howard, R. A.: Decision Analysis: Perspectives on Inference, Decision, and Experimentation, Proc. IEEE, 58, 5 (1970), pp. 632-643.

16) Guikema, S. D. and Pate-Cornell, M. E.: Bayesian Analysis of Launch Vehicle Success Rates, J. Spacecraft Rockets, 41, 1 (2004), pp. 93-102.

17) Deep, K. and Thakur, M.: A New Crossover Operator for Real Coded Genetic Algorithms, Appl. Math. Comput., 188, 1 (2007), pp. 895-911.

18) Deep, K. and Thakur, M.: A New Mutation Operator for Real Coded Genetic Algorithms, Appl. Math. Comput., 193, 1 (2007), pp. 211-230.

19) Deep, K., Singh, K. P., Kansal, M. L., and Mohan, C.: A Real Coded Genetic Algorithm for Solving Integer and Mixed Integer Optimization Problems, Appl. Math. Comput., 212, 2 (2009), pp. 505-518.

20) European Space Agency: Fact Sheet, 2015, Available: http: //www.spacetelescope.org/about/general/fact_sheet/ (accessed 201505-15)

21) National Aeronautics and Space Administration: James Webb Space Telescope (JWST): Independent Comprehensive Review Panel (ICRP) Final Report, Washington, DC, 2010.

22) British Broadcasting Corporation: EU Awards Galileo Satellitenavigation Contracts, 2010.

23) Planet Labs: Planet Labs Homepage, https://www.planet.com/ (accessed 2017-04-02)

24) The Times of India: ISRO Creats History, Launches 104 Satellites in One Go, 2017.
Jun'ichiro Kawaguchi Associate Editor 